\title{
MUJER Y DISCAPACIDAD: PERCEPCIÓN DE SU CALIDAD DE VIDA
}

\author{
María Victoria Martín-Cilleros', María Cruz Sánchez-Gómez¹, Miguel Ángel Verdugo-Alonso', \\ Manuela Crespo-Cuadrado', Ana Belén Sánchez-García', Cristina Caballo-Escribano', Sonia \\ Verdugo-Castro ${ }^{1}$ y Elena Manjón-García ${ }^{1}$ \\ ${ }^{1}$ Instituto Universitario de Integración en la Comunidad (INICO) Universidad de Salamanca, España. viquimc@usal.es \\ mcsago@usal.es.
}

\begin{abstract}
Resumen: Introducción: La discapacidad es una condición que afecta a la calidad de vida de las personas, si quien la padece es una mujer, esta afectación puede llegar a ser más limitante. Ante la escasa literatura y la necesidad de conocer la situación actual con fines de intervención, se realiza el siguiente estudio; Objetivos: Conocer la percepción de la mujer con discapacidad teniendo en cuenta aquellos aspectos que contribuyen a su desarrollo integral e inclusivo Métodos: Mediante un cuestionario de preguntas abiertas ad hoc se ha realizado un análisis cualitativo de los datos utilizando el programa NVivo 12; Resultados: La mujer del ámbito rural y urbano siente más preocupación por su autodeterminación e inclusión, ambas dimensiones destacan independientemente del tipo de discapacidad; mientras que aquellas que viven en lugares intermedios además de la autodeterminación les preocupa su desarrollo personal, derechos y bienestar físico. Se han encontrado diferencias según la edad, siendo el bienestar emocional una dimensión a tener en cuenta a medida que la mujer avanza en su ciclo vital. Conclusiones: Es necesario una mayor sensibilización de la población, así como facilitar tanto recursos como espacios que permitan a la mujer con discapacidad un mayor empoderamiento y participación realmente inclusiva.
\end{abstract}

Palabras clave: Discapacidad; Mujer; Bienestar, Calidad de Vida, Enfoque Cualitativo.

\section{WOMEN AND DISABILITY: PERCEIVING THEIR QUALITY OF LIFE}

\begin{abstract}
Introduction: Disability is a condition that affects the quality of life of people, if the one who is affected is a woman, this affectation can become more limiting. In view of the limited literature and the need to know the current situation for intervention purposes, the following study is carried out; Objectives: To know the perception of women with disabilities taking into account those aspects that contribute to their integral and inclusive development; Methods: A qualitative analysis of the data was carried out using the NVivo 12 programme by means of an ad hoc open questionnaire; Results: Rural and urban women are more concerned about their self-determination and inclusion, both dimensions stand out independently of the type of disability; while those who live in intermediate places besides self-determination are concerned about their personal development, rights and physical well-being. Differences have been found according to age, with emotional wellness being a dimension to take into account as the woman advances in her life cycle; Conclusions: It is necessary to raise awareness among the population, as well as to provide both resources and spaces that allow women with disabilities greater empowerment and truly inclusive participation.
\end{abstract}

Keywords: Disability; Woman; Welfare, Quality of Life, Qualitative Approach

\section{INTRODUCCIÓN}

La observación presentada por el Comité sobre los derechos de las Personas con discapacidad sobre las mujeres y las niñas con discapacidad (Comité de Naciones Unidas sobre los derechos de las personas con discapacidad, 2016), indica que existen pruebas sólidas que demuestran que las mujeres y las niñas con discapacidad afrontan obstáculos 
en la mayor parte de los ámbitos de vida y que dichos obstáculos generan situaciones en las que existen múltiples e interseccionales formas de discriminación contra ellas. Señala que estas en concreto tienen que ver con: la igualdad de acceso a la educación, las oportunidades económicas, la interacción social y la justicia, el igual reconocimiento como persona ante la ley y la capacidad de participar en la política y ejercer control sobre sus propias vidas en diversos contextos como el de atención a la salud, incluidos los servicios de salud sexual y reproductiva, y de decisión sobre dónde y con quién desean vivir.

Destaca así que las mujeres y las niñas con discapacidad tienen más probabilidad de ser objeto de discriminación que los hombres y los niños con discapacidad y que las mujeres y las niñas sin discapacidad.

El Comité señala además que las leyes y políticas internacionales sobre la discapacidad han desatendido históricamente los aspectos relacionados con las mujeres y las niñas con discapacidad y que también las leyes y políticas relativas a la mujer han hecho caso omiso de la discapacidad. Así considera que esto ha llevado a una situación de invisibilidad que se ha perpetuado en situaciones de formas múltiples e intersectoriales de discriminación contra las mujeres y las niñas con discapacidad que son a menudo objeto de discriminación por motivos de género y/o discapacidad y también por otros motivos.

Entre estos motivos, se alude a la edad, la discapacidad, el origen étnico, nacional o social, la identidad de género, la opinión política o de otra índole, la raza, la condición de migrante, refugiado o solicitante de asilo, la religión, el sexo y la orientación sexual.

De este modo, indica que las mujeres con discapacidad pueden encontrarse en situaciones en las que varios motivos o factores, como el sexo y la discapacidad, entre otros o junto con otros, interactúen al mismo tiempo, de forma que son inseparables y conducen a situaciones de discriminación complejas agravadas y específicas.

Destaca entonces que las mujeres con discapacidad son un grupo diverso en base a todas las variables anteriores y otras posibles, y advierte sobre la necesidad de tener esto en cuenta, pues en una mujer concreta la interacción de diversas de las variables descritas puede llevar a situaciones de discriminación aumentadas en base a tal conjunto complejo de interacciones (Comité de Naciones Unidas sobre los derechos de las personas con discapacidad, 2018). 
Varias voces sugieren que el hecho de que las mujeres con discapacidad superen a los hombres, sobre todo a medida que la edad aumenta, no deriva solo de la aparición de condiciones biológicas, sino más bien de la existencia de estas situaciones y experiencias de discriminación relacionadas con la imposibilidad de tomar decisiones sobre sus propias vidas, el paternalismo con el que conviven, la falta de información y la falta de autonomía en la toma de decisiones en su vida cotidiana (Gomiz, 2017).

Como se ha comentado previamente, la historia de las mujeres con discapacidad es también una historia de resiliencia. Ciertamente, a pesar de la existencia de situaciones discriminación, exclusión, ausencia de visibilidad, vulneración de derechos y violencia contra ellas, muchas de estas mujeres ya están organizadas en movimientos activos y decididas a luchar para defender sus derechos.

Del trabajo realizado por estos colectivos de mujeres han surgido muchas propuestas y planteamientos para avanzar en la mejora de sus condiciones de vida los cuales se detallan, entre otros, en el Segundo Manifiesto de los Derechos de las Mujeres y Niñas con discapacidad de la Unión Europea (Comité de Mujeres del Foro Europeo de la discapacidad, 2011) o, a nivel nacional, en el Segundo Plan Integral de Acción de Mujeres con Discapacidad 2013-2016 (CERMI, Fundación CERMI Mujeres, 2013).

Datos recientes proporcionados por el Observatorio Estatal de la Discapacidad (OED, 2018), indican que, según la Encuesta Nacional de Salud realizada en 2017, un 22,8\% de las personas residentes en España se han visto limitadas en los últimos seis meses para realizar las actividades de la vida cotidiana debido a un problema de salud. De nuevo, el porcentaje crece con la edad y es sustancialmente más alto entre las mujeres que entre los hombres.

En España, en el año 2008, según la Encuesta de Discapacidad, Autonomía Personal y Situaciones de Dependencia, EDAD, presentaban discapacidad más de tres millones y medio de personas. Casi el $60 \%$ de estas eran mujeres y los porcentajes de mujeres con discapacidad con respecto a los hombres se veían incrementados a medida que aumentaba la edad (INE, 2009).

En Castilla y León, en el año, en 2015 había un total de 169.637 personas con discapacidad, lo cual supone una prevalencia del 6,86\% sobre el total de la población. El 48,2\% de estas personas eran mujeres y el $51,8 \%$ varones. 
El perfil es similar al reflejado en los datos mundiales y españoles afectando sobre todo a las personas de entre 50 y 89 años (que suponen el 69\% de las personas con discapacidad de Castilla y León).

A partir de los 65 años y sobre todo de los 80 se produce también en Castilla y León una feminización de la vejez y las mujeres con discapacidad superan a los hombres. En concreto, para las discapacidades que generan mayor dependencia el número de mujeres duplica al de hombres. Finalmente, casi la mitad $(44,4 \%)$ de estas personas con discapacidad viven en entornos rurales con baja densidad de población o incluso despoblación lo que plantea la necesidad de crear prestaciones y recursos adecuados a las demandas concretas de personas con discapacidad que viven en zonas rurales dispersas (Consejería de familia e igualdad de oportunidades de Castilla y León, 2017).

Se considera de especial necesidad conocer la situación real de las mujeres con discapacidad al objeto de sensibilizar a la población y tener como base los resultados para emprender futuras acciones y acercarse a la situación de dicho colectivo.

\section{MÉTODO}

\subsection{Objetivos}

A través de una revisión amplia de bibliografía relacionada con el tema, se constata que no existen en la actualidad muchas investigaciones que recojan información sobre este colectivo en Castilla y León. De ahí, la importancia de los resultados de este estudio, cuyo objetivo principal es conocer la percepción de la mujer con discapacidad en Castilla y León teniendo en cuenta aquellos aspectos que contribuyen a su desarrollo integral e inclusivo. Este estudio es de especial interés, puesto que supone analizar las demandas de las mujeres con discapacidad y proporcionar sugerencias de mejora del sistema de dependencia.

\subsection{Procedimiento}

En situaciones como las planteadas en este estudio, en que los contextos a investigar están claramente definidos (mujeres con discapacidad de Castilla y León), y la población implicada en la intervención es limitada, un planteamiento meramente extensivo no permitiría la realización de un análisis minucioso como el que propone el enfoque intensivo. Por estas 
razones teórico-metodológicas y pragmáticas, se consideró pertinente la utilización de métodos cualitativos para identificar y proponer líneas de intervención.

Para el estudio se elaboró un cuestionario ad hoc de preguntas abiertas dirigido a conocer expectativas, creencias, percepciones y opiniones de las mujeres con discapacidad castellano-leonesas, el cual fue difundido a través de representantes, a instituciones oficiales y asociaciones de personas con discapacidad. Se realiza un muestro incidental de la población de la zona rural y urbana de la comunidad de Castilla y León, con respuesta de 526 personas, ofreciendo la información las propias interesadas, cuidadores primarios 0 tutores.

Una vez volcada toda la información en el programa CAQDAS Nvivo 12, desde una plantilla de Excel, se procedió a la categorización y codificación o asignación del espacio textual a la categoría correspondiente (Sánchez, Delgado y Santos, 2013).

Previo al proceso de categorización, se realizó un análisis global que consistió en: a) reuniones del equipo para clarificar conceptos clave, en nuestro caso apoyos recibidos y su relación con la calidad de vida de las mujeres con discapacidad, b) antes de la codificación se leyó varias veces el texto para detectar afirmaciones centrales, anotar las ideas y palabras clave; y, sus posibles definiciones.

El proceso se completó realizando juicios de valor sobre este análisis, lo que permitió elaborar un libro de códigos para que los codificadores no cometieran errores al asignar texto a una categoría correspondiente. Los análisis de respuestas se realizaron mediante un análisis de contenido clásico siguiendo las fases: reducción, disposición y transformación de los datos utilizando una herramienta informática para facilitar el trabajo (CAQDAS Nvivo 12).

\subsection{Resultados}

Posteriormente, y siguiendo los criterios para garantizar la calidad en la investigación cualitativa (Palacios, Gutiérrez y Sánchez 2013), la codificación fue realizada bajo la supervisión del equipo, para así poder garantizar la credibilidad del proceso. 


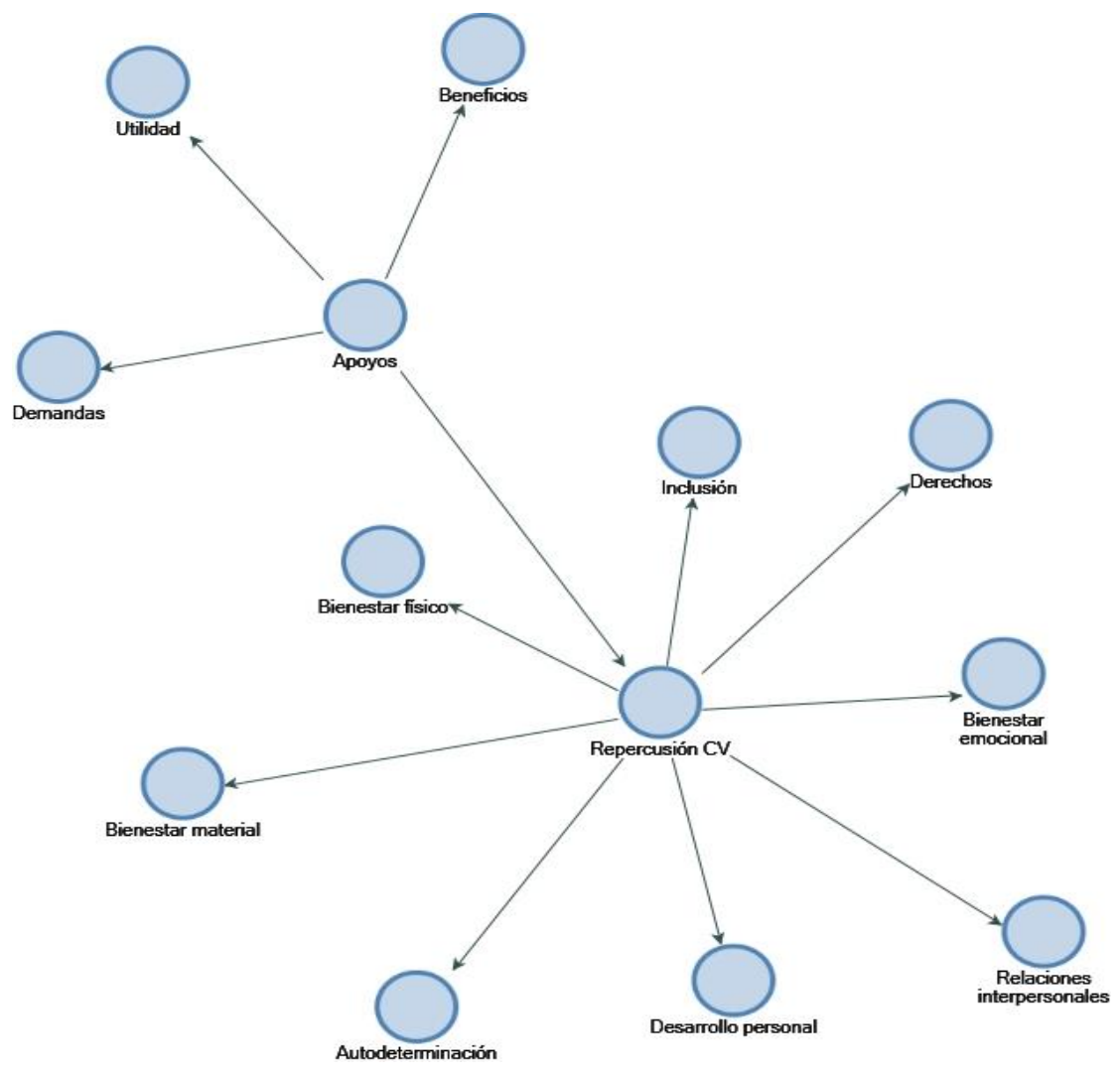

Figura 1. Mapa conceptual de categorías.

De acuerdo a los comentarios ofrecidos, surge el mapa conceptual (ver figura 1) que está centrado en los apoyos. Sus categorías quedan definidas de forma exhaustiva y contextualizada por demandas, utilidad, beneficios y repercusión en la calidad de vida (CV), a partir de la cual se distinguen las subcategorías correspondientes a las ocho dimensiones propuestas por Schalock y Verdugo (2003).

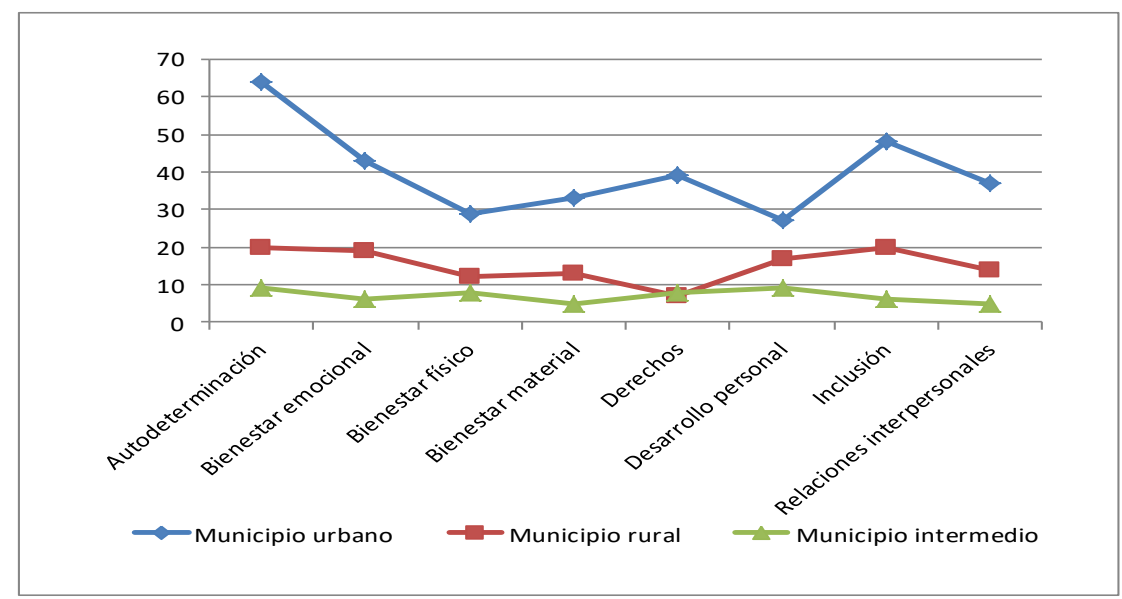

Figura 2. Frecuencia de comentarios ofrecidos según dimensión de calidad de vida y lugar de residencia. 
Haciendo un análisis más profundo sobre la variable lugar de residencia (ver figura 2), se observa como aquellas mujeres que viven en el medio urbano, han sido más participativas en la encuesta de opinión. Dichas mujeres, al igual que las que viven en medios rurales manifiestan más comentarios sobre la autodeterminación y la inclusión, ocupando esta segunda variable un quinto puesto para mujeres que viven en municipios intermedios, aquellos municipios entre 10.000 y 50.000 habitantes. Las mujeres de estos últimos municipios ofrecen mayores comentarios, además de en autodeterminación y desarrollo personal, en derechos y bienestar físico.

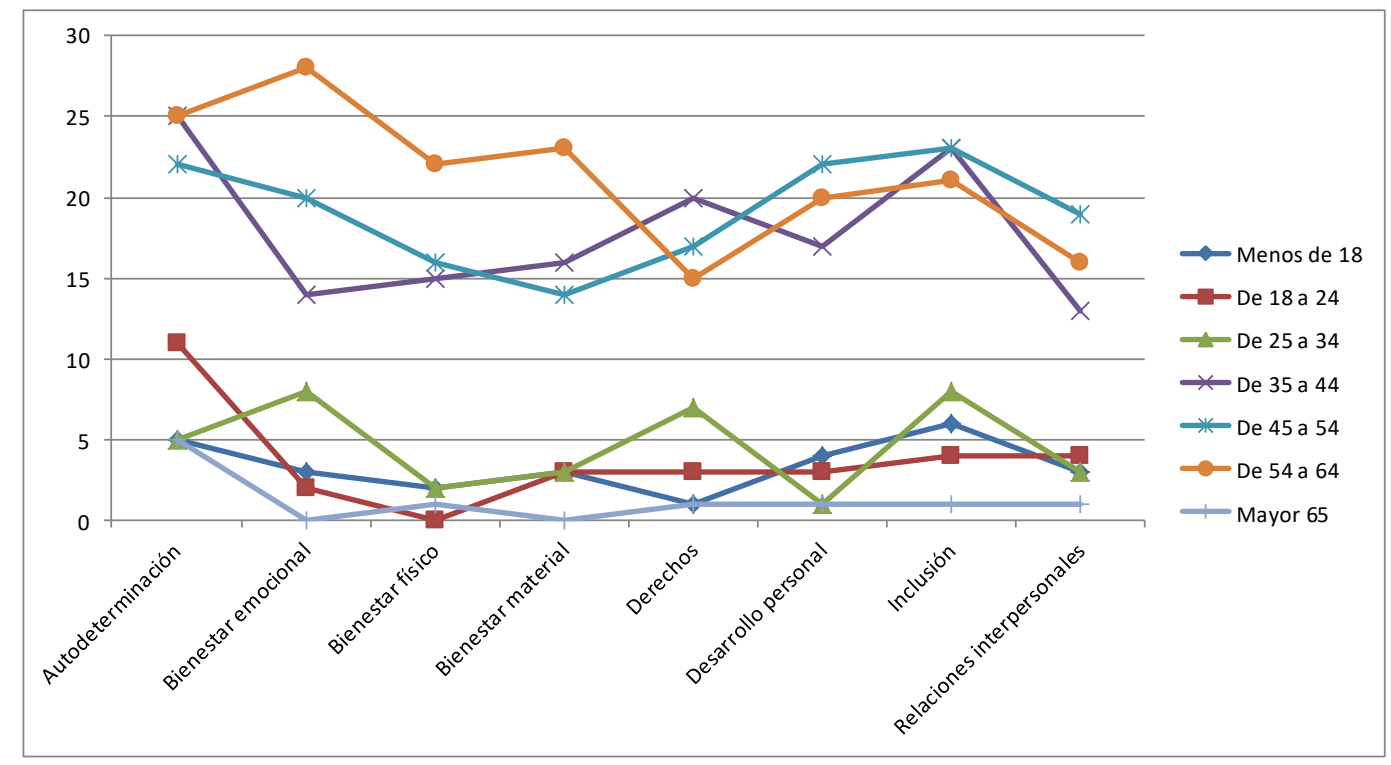

Figura 3. Frecuencia de comentarios ofrecidos según dimensión de calidad de vida y edad.

Como se puede apreciar en la figura 3, los comentarios han variado según el rango de edad, siendo más frecuentes en el rango de edad entre 35 y 64 años con preocupaciones diferentes a las de un rango menor. Se observa que en mujeres con menos de 18 años el tema más comentado es la inclusión, la autodeterminación y el desarrollo personal, prestando menos interés a los derechos o el bienestar físico. Manifiestan comentarios donde se han sentido excluidas "la expulsaron de un curso de natación", "se siente excluida porque necesita que le expliquen las cosas de distinta manera y decide no participar"; también hay comentarios de que se han sentido dependientes tanto físicamente como para mejorar su desarrollo personal "dependo de otras personas para muchas actividades", "uso silla de ruedas y no puedo caminar y ello me impide poder entrar en muchos sitios y tengo necesidad de que me ayude otra persona si utilizo aseos". 
Cuando se ha preguntado a mujeres entre los 18 y 34 años la inclusión pasa a un segundo plano siendo más relevante la autodeterminación, y cogiendo más interés posteriormente el bienestar emocional y los derechos. Nos encontramos con comentarios de mujeres entre los 18 y 24 años que se sienten totalmente dependientes "dependo absolutamente de mis padres", "soy totalmente dependiente"; manteniéndose esta situación entre los 25 y 35, con comentarios como "total y absoluta, siempre me han ordenado lo que tenía que hacer, cuándo y de qué manera", "que no me gustan que decidan por mí, me considero divertida e independiente y no me dejan ser como yo quiero". La falta de inclusión, como manifiesta una mujer entre los 18 y 24 años, le ha afectado también al bienestar emocional "a veces he pasado malas temporadas por culpa de discriminación por mi discapacidad" o a las relaciones interpersonales "nunca he podido participar en este tipo de actividades por no encontrar un grupo con quién hacerlas o no ser invitada para ello ".

Las mujeres entre los 25 y 34 años comentan que esta situación de sensación de discriminación sigue en la siguiente franja de edad: "hace que todos los de alrededor tiendan a ayudarte y ocuparse de ti entonces ya no eres una más", "evito a todas aquellas personas que dándoles unas pautas básicas para que la comunicación sea más fácil las ignoran, y aquellas que se lo toman como algo gracioso ".

En relación al bienestar emocional, las mujeres menores de 18 años comentan sentimientos de baja autoestima, acoso o inseguridad que duran hasta los 24 años "La autoestima la tienen muy baja porque piensan que todo lo hace mal", "ha sufrido bulling en el colegio y en el comedor, otros niños se ríen de ella y hacen ruidos y cantan que saben que le molesta; se mofan muy a menudo"; a partir de los 25 , incluso un poco antes, en algunas participantes comienzan los problemas de depresión "he sufrido y sufro depresión desde hace casi tres años, estoy en tratamiento con una psiquiatra; he conseguido recuperarme bastante, pero no del todo", "me encuentro deprimida", "cuando me operaron me afectaron a nivel emocional los cambios físicos transitorios que se originaron" (comentarios de mujeres entre los 25 y 34). Estos síntomas van a estar más presentes entre los 35 y los 44 , con diagnósticos de depresión, ansiedad "va, a psicólogo para ser fuerte", "a veces tengo bajones y necesito hablar con el psicólogo del centro y contarle mis cosas", "con depresiones temporales".

Finalmente, entre 35 y 65 existen comentarios en todas las dimensiones, destacando de nuevo ligeramente la autodeterminación y la inclusión. 
Las mujeres entre los 35 y 44 años especifican más los temas con los que consideran que no tienen autodeterminación siendo el más recurrente los problemas con el lugar de residencia: "no he elegido dónde ni con quién vivir", "no puedo elegir lo que quiero no puedo elegir donde vivir", "no poder vivir sola", "todavía vivo en mi casa con mis padres y hermanos". En el intervalo de los 45 a los 54 describen esa situación de dependencia desde el pasado "han elegido por mi mucha cosa como el lugar de residencia por ejemplo", "no viajaba sola, me daba miedo; siempre me acompañaba mi madre o hermanas"; y manteniéndose aún hoy día "no puedo ir sola en el autocar hasta mi pueblo; mi familia no me lo autoriza", "me gustaría hacer más actividades", "no puedo disponer de mi dinero", "no puedo realizar todas las actividades que deseo, como viajar a lugares determinados"; en algunos casos es la propia discapacidad la que limita la autodeterminación "que quiero hacer más de lo puedo yo".

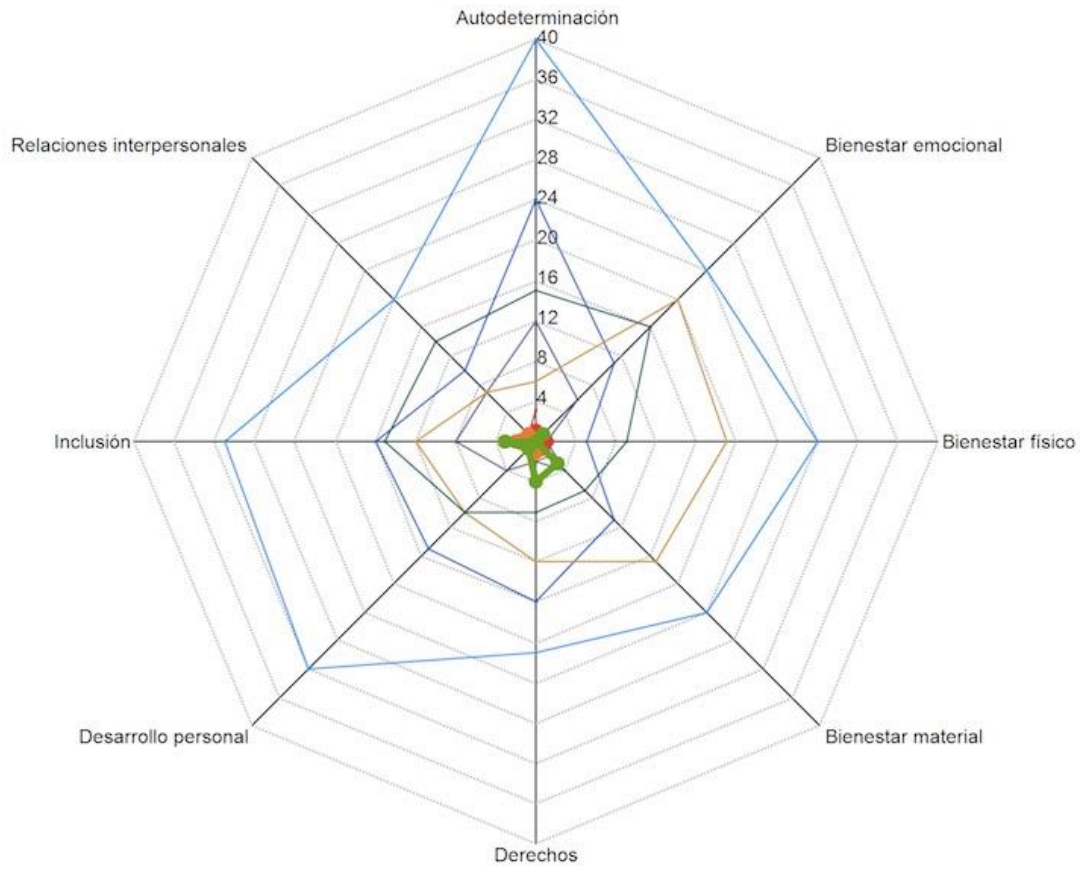

Discapacidad intelectual N/A

Otras

Parálisis cerebral

Discapacidad Fisica

Salud Mental

Multidiscapacidad

Daño cerebral

Enfermedades raras

Discapacidad visual

Discapacidad auditiva TEA

Figura 4. Frecuencia de comentarios ofrecidos según dimensión de calidad de vida y tipo del discapacidad.

Cuando los comentarios se analizan de acuerdo al tipo de discapacidad, las dimensiones más comentadas hacen referencia a la autodeterminación e inclusión, siendo los temas menos abordados las relaciones interpersonales y el bienestar físico. 
Las mujeres manifiestan limitaciones en su autodeterminación de acuerdo a sus opiniones, de las cuales aproximadamente un $40 \%$ fueron a cargo de personas que presentaban discapacidad múltiple, donde reflejan experiencias relacionadas con la total dependencia de los padres "dependo totalmente de mis padres", seguido de un $24 \%$ por personas con discapacidad intelectual, donde los comentarios hacen referencia a no poder elegir en diferentes aspectos de sus vida "no he elegido dónde ni con quién vivir", "no siempre se respetan mis decisiones "; un $15 \%$ de personas con problemas de salud mental que abarcan los dos aspectos comentados anteriormente con manifestaciones del tipo "no se administrar el dinero; me tienen que ayudar y a veces gasto todo lo que tengo en objetos que no necesito", "tener derecho a la autodeterminación, tomar nuestras propias decisiones"; y un 12\% aquellas que presentan parálisis cerebral que se enfocan más en la total dependencia que tienen de un apoyo "total y absoluta, siempre me han ordenado lo que tenía que hacer, cuándo y de qué manera", "constante vigilancia". Esta dimensión fue menos tenida en cuenta por las personas con alguna discapacidad física o enfermedad rara.

En los comentarios relacionados con la dimensión de inclusión se percibe una sensación de rechazo generalizada: "me he sentido discriminada cuando he acudido al médico"; donde no se le reconocen derechos en el trabajo e intimidad: "es mucho más difícil cuando se tiene una discapacidad encontrar un trabajo; a mí me gustaría trabajar y encontrar un trabajo estable", "me gustaría tener más intimidad".

\section{CONCLUSIONES}

De acuerdo a los comentarios manifestados por las mujeres podemos concluir que presentan una afectación en todas las dimensiones de la calidad de vida. Hacen un mayor énfasis en la falta de autonomía, con una constante sensación de dependencia, y falta de elección, debiendo amoldarse a sus cuidadores o tutores. Aunque la presencia es cada vez mayor en la comunidad, la participación continúa siendo una asignatura pendiente, bien por las barreras arquitectónicas o por las más difíciles de eliminar, como son las mentales. El círculo de amistades es reducido, siendo difícil la creación de nuevas redes sociales debido a las dificultades que encuentran para aumentar la participación en la comunidad, como se comentó anteriormente y por la pérdida de ellas cuando la discapacidad aparece en la vida de la persona o la familia.

En el desarrollo personal los comentarios suelen ahondar más en el ámbito profesional, donde es difícil encontrar trabajo o incluso la discapacidad lleva a una situación de 
desempleo. Estas situaciones hacen que el bienestar material se vea muy afectado, unido a los gastos derivados de la discapacidad por temas de salud, arquitectónicos u otros tipos de apoyos.

Aunque no haya sido una dimensión muy comentada, el bienestar físico está condicionado igualmente a su situación de dependencia, siendo un colectivo con bastantes problemas de salud algunos de ellos efectos secundarios de la medicación. Entre los problemas de salud, la salud mental está muy afectada, cuanto mayor es la edad de la mujer mayor afectación.

Al igual que otros estudios han venido observando (Gomiz, 2017; Shu y Conde, 2009), las participantes manifiestan problemas emocionales, tales como baja autoestima, no siendo abordados por profesionales hasta que no derivan en depresión u otros problemas de salud mental más serios. Es necesario que este apoyo emocional esté presente desde los inicios de la manifestación de la enfermedad cuando esta surge en el desarrollo evolutivo de la persona y que se trabaje este aspecto desde etapas infantiles cuando la persona presenta la discapacidad desde el nacimiento. Este aspecto es eclipsado, a la vez que potenciado, por sus problemas económicos, puesto que una de las principales necesidades que consideran no cubiertas tiene que ver con los gastos que se derivan de su discapacidad, como son los gastos médicos, dirigidos a apoyos físicos o arquitectónicos. Estas necesidades básicas hacen que no dispongan de ingresos para otras actividades de tipo lúdico, que junto con la discriminación que encuentran en la comunidad en el momento de la participación, y en algunas circunstancias, una autoimagen negativa, inciden en el bienestar emocional, siendo muy habitual en los comentarios "tengo depresión", "ansiedad".

De los resultados se desprende la necesidad de continuar trabajando la independencia y autonomía, viéndose la figura del apoyo personal como algo muy beneficioso tanto para la persona con discapacidad que le permite mayor autonomía y planificación, como para los familiares que les sirve de respiro. Actualmente esta figura solo se utiliza para las necesidades básicas como vestido o acompañamiento a centros médicos, pidiendo una mayor disponibilidad de los mismos, tanto en número como en horas. Los comentarios indican la necesidad de más asistentes personales, regulados por la administración y con una formación determinada. Igualmente necesario es la sensibilización de la población en general, facilitando más espacios de participación delineados bajo la orientación de diseño universal. 
Agradecimientos. Agradecemos al Comité Autonómico de Entidades de Representantes de Personas con Discapacidad (CERMI) en Castilla León la financiación del estudio, y a todas las mujeres que han compartido con el/las autor/as sus inquietudes.

\section{REFERENCIAS}

CERMI, Fundación CERMI Mujeres (2013). II Plan Integral de Acción de Mujeres con discapacidad 2013-2016. Madrid: CINCA.

Comité de Mujeres del Foro Europeo de la Discapacidad (2011). 2 Manifiesto de los derechos de las Mujeres y Niñas con Discapacidad de la Unión Europea. Una herramienta para activistas y responsables políticos. Vitoria-Gasteiz: EMAKUNDE-Instituto Vasco de la Mujer.

Comité sobre los derechos de las personas con discapacidad de Naciones Unidas (2016). Observación general número 3 sobre las mujeres y las niñas con discapacidad. Naciones Unidas: Convención sobre los derechos de las Personas con Discapacidad.

Comité sobre los derechos de las personas con discapacidad de Naciones Unidas (2018). Observación general número 6 sobre la igualdad y la no discriminación. Naciones Unidas: Convención sobre los derechos de las Personas con Discapacidad.

Consejería de familia e igualdad de oportunidades de Castilla y León (2017). Acuerdo 7/17 del 9 de febrero de la Junta de Castilla y León, por el que se aprueba el plan estratégico de igualdad de oportunidades para las personas con discapacidad 2016/2020. Boletín Oficial de Castilla y León BOCYL, 29. 4189-4268.

Gomiz, P. (2017). Vulnerabilidad y exclusión social en las mujeres con discapacidad. Documentación social, $184,153-178$.

Instituto Nacional de Estadística, INE (2009). Panorámica de la discapacidad en España. Encuesta de Discapacidad, Autonomía personal y situaciones de dependencia, 2008. Boletín Informativo del Instituto Nacional de Estadística, 1-12

Observatorio Estatal de la Discapacidad OED (2018). Personas con discapacidad y discriminación múltiple. Recuperado $14 \quad$ octubre 2019: https://observatoriodeladiscapacidad.info/attachments/article/102/DISCRIMINACION\%20MULTIPLE\%200 ED.pdf

Palacios, B., Gutiérrez, A., \& Sánchez, M. C. (2013). NVIVO 10: Una herramienta para la investigación en Comunicación. In M. Pacheco, M. Vicente, \& T. González (Eds.), Investigar la Comunicación hoy. Revisión de políticas científicas y aportaciones metodológicas. Simposio Internacional sobre Política Científica en Comunicación (Vol. 4, pp. 1003-1018).

Sánchez-Gómez, M.C., Delgado; M.C., y Santos, M.C. (2013). El proceso de la investigación cualitativa. Manual de procedimiento: ejemplificación con una tesis doctoral. Valladolid: Edintras.

Schalock, R. L., y Verdugo, M. A. (2007). Calidad de vida: manual para profesionales de la educación, salud y servicios sociales. Madrid: Alianza.

Shum, G. y Conde, A. (2009). Género y discapacidad como moduladores de la identidad. Feminismos, 13, 119132. 